\title{
A 19-year-old woman with pleuritic chest pain
}

\author{
Faisal A Khasawneh MD ${ }^{1}$, Mansoor Mehmood MD ${ }^{1}$, Ruba A Halloush MD²
}

\section{CASE PRESENTATION}

A 19-year-old woman with a medical history significant for poorly controlled diabetes mellitus (DM) (hemoglobin A1C 11.2\%) presented with a 12-day history of right-sided pleuritic chest pain. One month earlier, she had developed sudden-onset fever, dry cough and shortness of breath. At the time, she did not have any gastrointestinal symptoms including epigastric pain, heartburn, vomiting or retching. She denied sick contacts and had no preceding symptoms of an upper respiratory tract infection. She was seen at a local emergency department and was released home on a 10-day course of oral doxycycline (100 mg every $12 \mathrm{~h}$ ) to treat a community-acquired pneumonia (CAP). After an initial two-week improvement, her complaints relapsed, with fevers, dry cough, poor appetite and the above-described chest pain. The patient did not smoke and she denied alcohol abuse or illicit drug use.

On presentation to the hospital, she was febrile (temperature $38.2^{\circ} \mathrm{C}$ ), her oxygen saturation was $86 \%$ on room air and she was thin. She was awake and oriented but in obvious pain. She had no jugular venous distention. Her heart sounds were regular without murmurs or gallops. The lung examination revealed reduced air entry over the right lung base with bronchial breath sounds and egophony. The abdomen was soft without tenderness or distension and she had no evidence of subcutaneous emphysema or lymphadenopathy. There were no lower limb edema and no joint swelling or deformities.

The laboratory tests revealed a white blood cell count of $26.0 \times 10^{9} / \mathrm{L}$ and a hemoglobin level of $119 \mathrm{~g} / \mathrm{L}$. Blood chemistry showed normal kidney and liver function. Tests for HIV and pregnancy were negative. Chest imaging included a chest $\mathrm{x}$-ray and a contrasted chest computed tomography scan (Figures 1 and 2). The patient was admitted to hospital with severe sepsis due to CAP complicated by an empyema and was started on intravenous broad-spectrum antibiotics, vancomycin ( $15 \mathrm{mg} / \mathrm{kg}$ every $12 \mathrm{~h}$ ) and piperacillin/tazobactam (3.375 g every $6 \mathrm{~h})$. She underwent computed tomography-guided drainage $48 \mathrm{~h}$ afterwards. An interventional radiologist performed the procedure under strict sterile techniques. Cytopathology (Figure 3) and cultures were ordered on the drained fluid.

What is your diagnosis?

\section{DIAGNOSIS}

The fluid Gram stain was negative and the culture showed pure growth of Candida albicans. Hematoxylin and eosin-stained section of the pleural

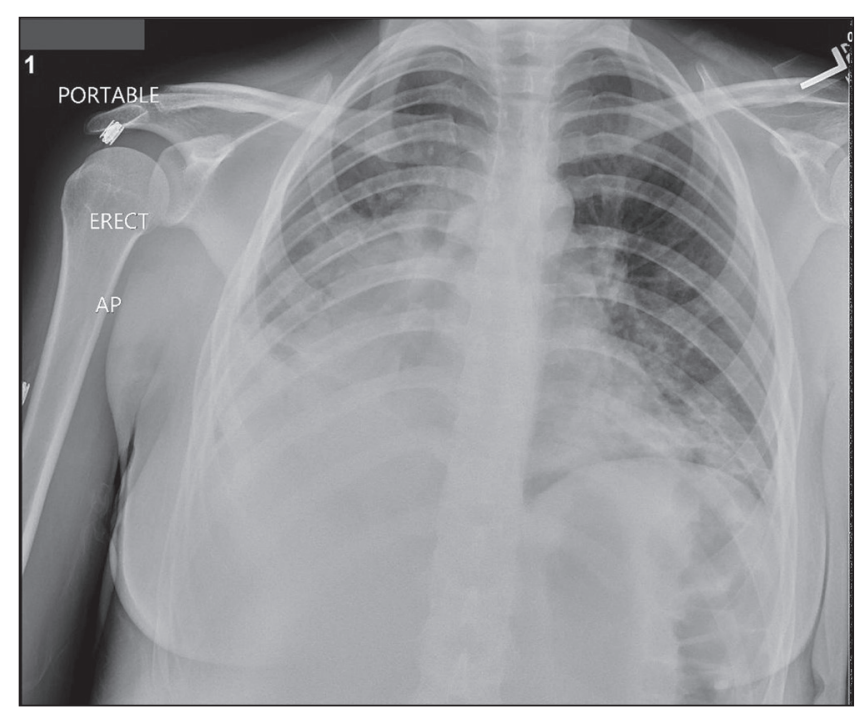

Figure 1) Chest $x$-ray showing dense right-sided consolidation

fluid showed numerous neutrophils and cellular debris consistent with empyema with occasional pseudohyphae. No squamous cells or ingested material was identified.

Based on the cytopathology findings and the aforementioned culture results, the patient was diagnosed with candida empyema thoracis (CET) that complicated an inadequately treated CAP with parapneumonic effusion. CET has been reported to be a part of a mixed infection in up to $46 \%$ of cases (1). A second pleural fluid sample was sent from the drain and it, once again, grew $\mathrm{C}$ albicans. She was started on intravenous micafungin (100 mg daily) with significant subsequent clinical improvement.

In the present case, we speculate that doxycycline disrupted the normal flora and allowed for candidal overgrowth. The patient's poorly controlled DM facilitated translocation from mucus surfaces into the blood stream and, subsequently, into the parapneumonic effusion, worsening the patient's symptoms $(2,3)$.

Before discharge, the intravenous antifungal was changed to oral fluconazole (200 mg daily) and her antibacterial therapy was de-escalated

${ }^{1}$ Section of Infectious Diseases, Department of Internal Medicine, Texas Tech University Health Science Center; ${ }^{2}$ Amarillo Pathology Group, Amarillo, Texas, USA

Correspondence: Dr Faisal A Khasawneh, Section of Infectious Diseases, Department of Internal Medicine, Texas Tech University Health Science Center, 1400 South Coulter Street, Amarillo, Texas 79106, USA. Telephone 806-414-9110, fax 806-354-5764,

e-mail faisal.khasawneh@ttuhsc.edu 


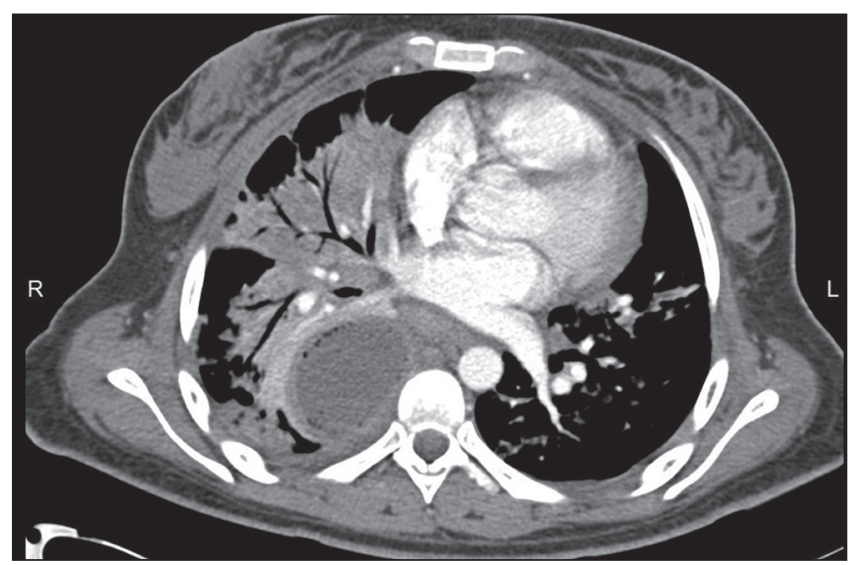

Figure 2) Computed tomography scan of the chest showing consolidation of the right lower and middle lobes with loculated effusion that has an enhancing rim and gas bubbles. There was no evidence of periesophageal fluid collections, esophageal thickening or mediastinal air

to oral moxifloxacin (400 mg daily). She was discharged home to finish a four-week course of the above therapy. She improved markedly and continues to be followed in the outpatient clinic.

\section{DISCUSSION}

CET is a rare entity. Most cases are acquired in health care settings (4). Community-acquired CET comprises $<15 \%$ of reported cases (4). Risk factors for this infection include malignancies, especially of the esophagus and stomach; DM; cirrhosis; and immunocompromised states including the use of long-term steroid therapy, HIV infection or having undergone organ transplantation $(1,4)$. Only a minority of individuals with this ailment have no underlying comorbidity. The infection results from direct inoculation into the pleural space following esophageal rupture, or after thoracentesis or thoracotomy. The former mechanism of infection is the most common $(1,4)$. On the other hand, it can spread from contiguous foci such as a fungal lung infection or a subdiaphragmatic abscess that complicated visceral perforation. On occasion, CET can result from hematogenous seeding. Fungemia was documented in 5\% to $20 \%$ of patients with this entity in the two largest cases series in the literature $(1,4)$.

C albicans is the most commonly implicated culprit causing fungal empyema, followed by Candida tropicalis and Candida glabrata $(1,4)$. On rare occasions, other fungi, such as Aspergillus and Cryptococcus, have also been implicated in causing this infection.

Patients with CET complain of fever, shortness of breath, cough and chest pain. They may also complain of the manifestations of the underlying condition that has led to the CET. For example, patients with esophageal rupture may complain of vomiting, severe chest pain and subcutaneous emphysema (5). Furthermore, patients' presentation may be complicated by florid sepsis with significant leukocytosis, organ failure and shock.

Diagnostic criteria for candida/fungal empyema thoracis include (4):

- Fungal isolation from exudative pleural effusion;

- Signs of active infection such as fever and leukocytosis;

- Isolating the same fungus from pleural effusion in more than one occasion, or from pleural effusion and another specimen such as blood or tissue cultures.

The latter point is not needed to confirm the diagnosis if the fungus has been cultured from a specimen that was appropriately collected from a sterile site (6). Furthermore, new markers of invasive fungal infection (ie, beta-D glucan and galactomannan) can be used to corroborate the diagnosis. Candida isolated from previously placed chest

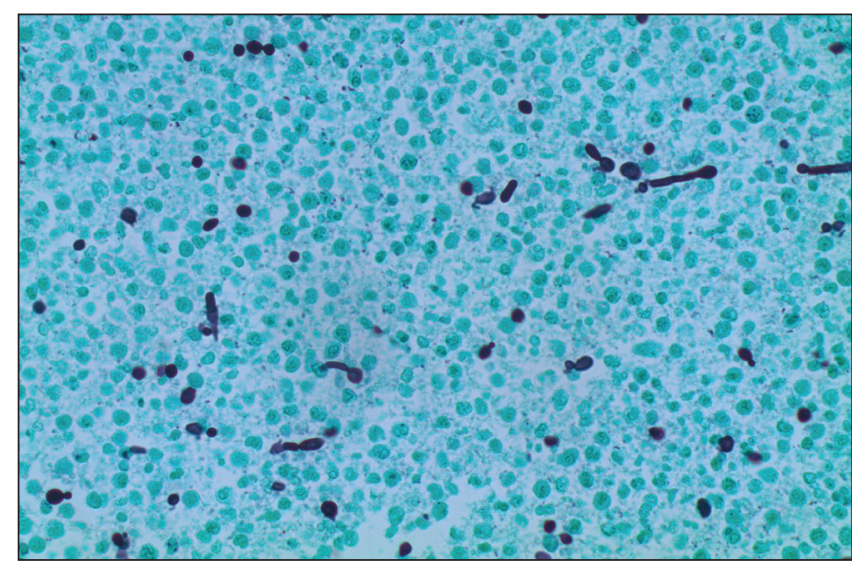

Figure 3) Gomori methanamine silver stain showing budding yeast and pseudohyphae consistent with Candida (original magnification $\times 400$ )

tubes or drains is considered a to be colonizer, unless infectious symptoms and signs persisted until antifungal therapy was initiated (1).

Early aggressive therapy is imperative to optimize outcome $(1,4)$. It consists of empyema drainage and antifungal therapy. The initiation of broad-spectrum antibiotic therapy pending culture results is indicated, given that approximately one-half of fungal empyema thoracis cases have a bacterial coinfection (1). It is important to note that the concentration of antifungal agents in pleural fluid is variable (1). Voriconazole and micafungin have been reported to provide good pleural penetration (7-9). On the other hand, anidulafungin and liposomal amphotericin B are reported to have poor pleural concentrations (10-12). Data regarding fluconazole pleural concentration are lacking; however, it has been used successfully alongside empyema drainage in the treatment of the majority of patients in the abovementioned case series $(1,4)$.

The prognosis of CET is guarded, with reported mortality rates as high as 60 to $70 \%(1,4)$. Being immunocompromised, developing acute respiratory failure or developing septic shock are risk factors for poor outcome.

\section{CONCLUSION}

CET is an uncommon invasive candidiasis. Predisposing risk factors include malignancies, DM, cirrhosis and immunocompromised states. The prognosis of this infection is grim unless prompt drainage and effective antimicrobial therapy are initiated in a timely manner.

DISCLOSURES: The authors have no conflicts of interest to declare.

\section{REFERENCES}

1. Lin KH, Liu YM, Lin PC, et al. Report of a 63-case series of Candida empyema thoracis: 9-year experience of two medical centers in central Taiwan. J Microbiol Immunol Infect 2014:47:36-41

2. Chuang TY, Yeh CY, Ko SW, Lin CJ, Lee SW, Hsueh PR. Fatal case of community-acquired empyema thoracis and candidemia caused by Candida albicans. Diagn Microbiol Infect Dis 2011;71:156-8.

3. Baradkar VP, Mathur M, Kulkarni SD, Kumar S. Thoracic empyema due to Candida albicans. Indian J Pathol Microbiol 2008;51:286-8

4. Ko SC, Chen KY, Hsueh PR, Luh KT, Yang PC. Fungal empyema thoracis: An emerging clinical entity. Chest 2000;117:1672-8.

5. Kim-Deobald J, Kozarek RA. Esophageal perforation: An 8-year review of a multispecialty clinic's experience. Am J Gastroenterol 1992;87:1112-9. 
6. Ishiguro T, Takayanagi N, Ikeya T, et al. Isolation of Candida species is an important clue for suspecting gastrointestinal tract perforation as a cause of empyema. Intern Med 2010;49:1957-64.

7. Matsuda T, Koreeda Y, Mataki H, Taira T, Noma S, Higashimoto I. A case of Aspergillus empyema successfully treated with combination therapy of voriconazole and micafungin: Excellent penetration of voriconazole and micafungin into pleural fluid. Intern Med 2010;49:1163-9.

8. Lin PY, Jean SS, Ou TY, Chen FL, Lee WS. Successful salvage therapy with micafungin for Candida empyema thoracis. J Microbiol Immunol Infect 2014 February 3 (Epub online ahead of print).

9. Stern JB, Girard P, Caliandro R. Pleural diffusion of voriconazole in a patient with Aspergillus fumigatus empyema thoracis.

Antimicrob Agents Chemother 2004;48:1065.
10. Moriyama B, Ditullio M, Wilson E, et al. Pharmacokinetics of anidulafungin in pleural fluid during the treatment of a patient with Candida empyema. Antimicrob Agents Chemother 2011;55:2478-80.

11. Moriyama B, Torabi-Parizi P, Pratt AK, et al. Pharmacokinetics of liposomal amphotericin B in pleural fluid. Antimicrob Agents Chemother 2010;54:1633-5.

12. Weiler S, Bellmann-Weiler R, Joannidis M, Bellmann R.

Penetration of amphotericin B lipid formulations into pleural effusion. Antimicrob Agents Chemother 2007;51:4211-3. 


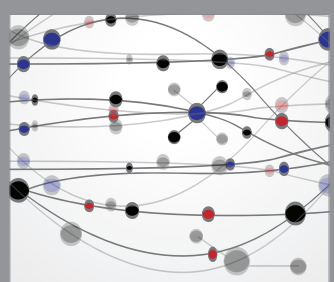

The Scientific World Journal
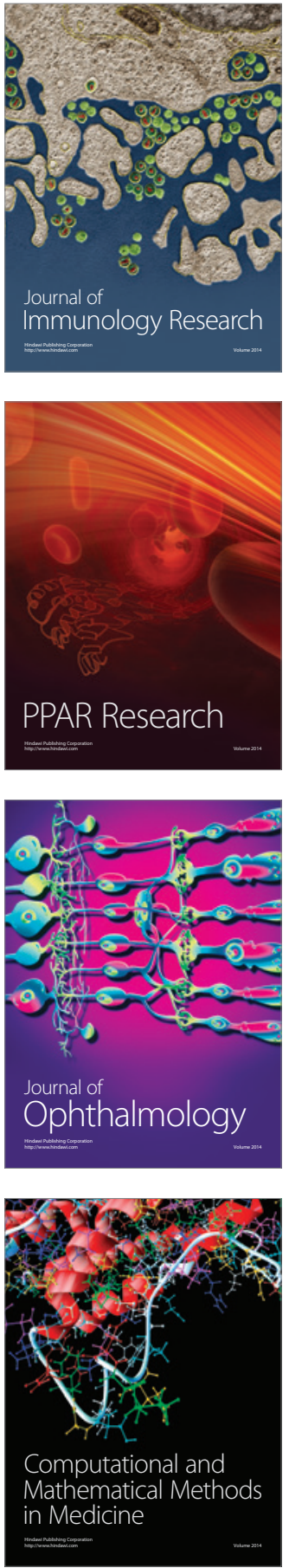

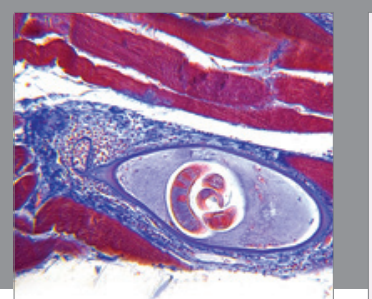

Gastroenterology Research and Practice

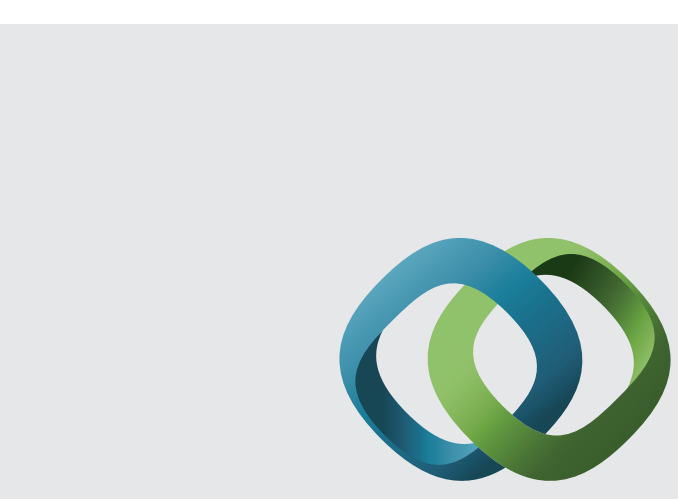

\section{Hindawi}

Submit your manuscripts at

http://www.hindawi.com
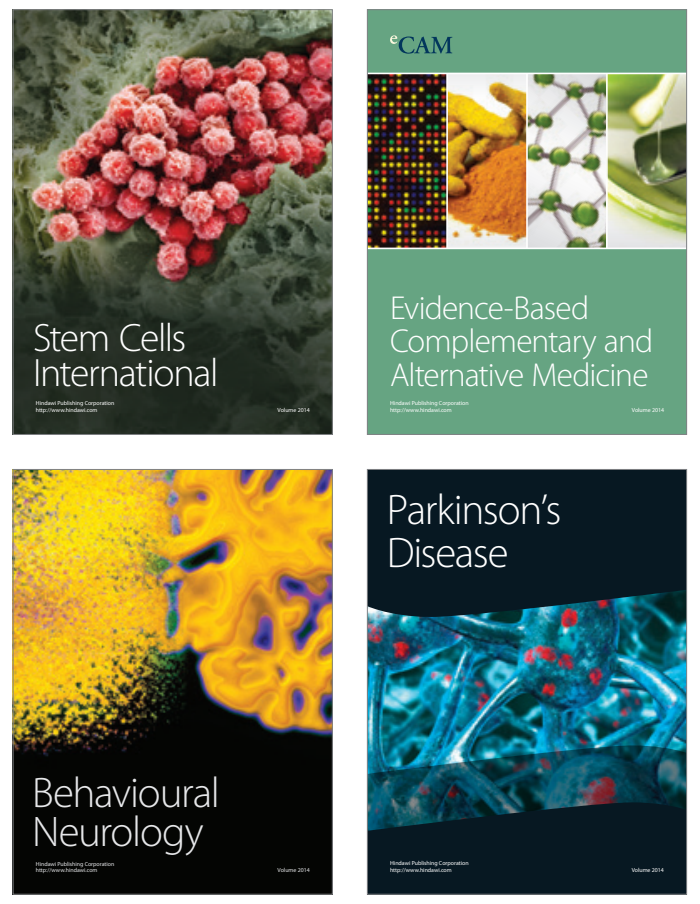
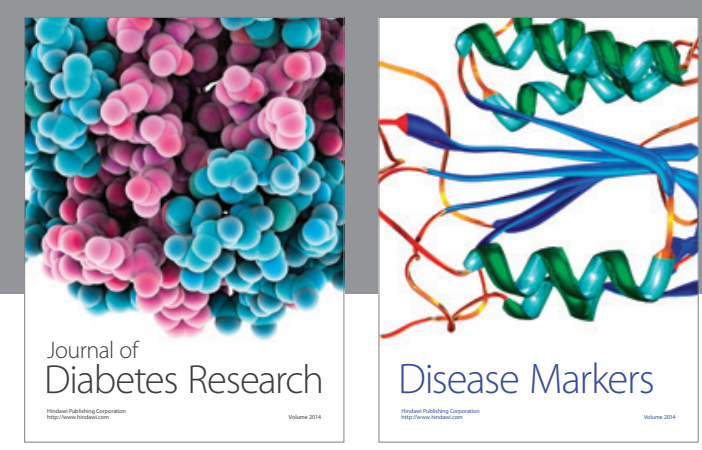

Disease Markers
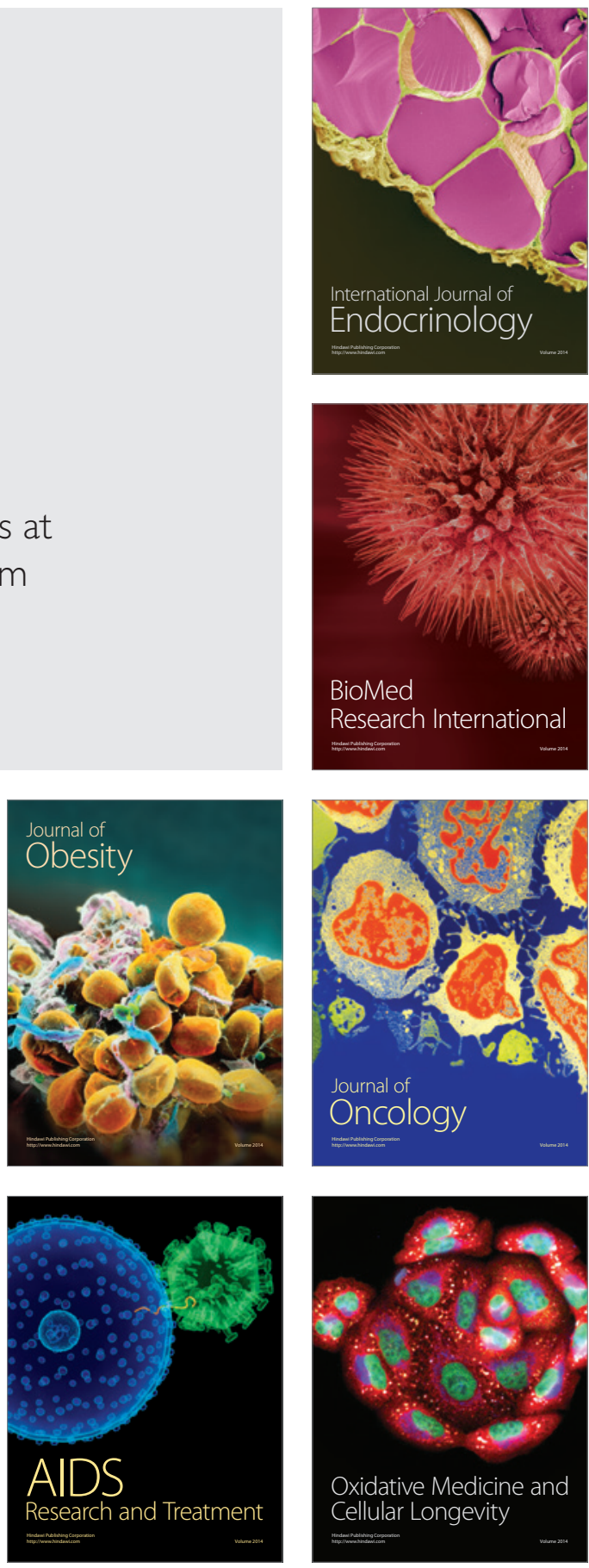